\title{
Multiple-Dose Pharmacokinetics of Ozanimod and its Major Active Metabolites and the Pharmacodynamic and Pharmacokinetic Interactions with Pseudoephedrine, a Sympathomimetic Agent, in Healthy Subjects
}

\author{
Jonathan Q. Tran (D) P Peijin Zhang · Susan Walker · Atalanta Ghosh • \\ Mary Syto $\cdot$ Xiaomin Wang · Sarah Harris · Maria Palmisano
}

Received: August 7, 2020 / Accepted: September 5, 2020 / Published online: October 6, 2020

(C) The Author(s) 2020

\begin{abstract}
Introduction: The aims of this study were to characterize the multiple-dose pharmacokinetics (PK) of ozanimod's major active metabolites (CC112273 and CC1084037) and to evaluate the pharmacodynamic and PK interactions with pseudoephedrine (PSE).

Methods: In this phase 1, single-center, randomized, double-blind, placebo-controlled study, 56 healthy adult subjects were randomized to receive either placebo or ozanimod once daily for 30 days $(0.23 \mathrm{mg}$ on days $1-4,0.46 \mathrm{mg}$
\end{abstract}

Digital Features To view digital features for this article go to https://doi.org/10.6084/m9.figshare.12918965.

J. Q. Tran (ه) · P. Zhang · M. Syto · M. Palmisano Clinical Pharmacology and Pharmacometrics and Research and Early Development, Bristol-Myers Squibb Company, Princeton, NJ, USA

e-mail: dr.jtran@gmail.com

S. Walker

Apex Biostatistics, Inc, New Hill, NC, USA

A. Ghosh

Biometrics and Data Sciences, Bristol-Myers Squibb

Company, Princeton, NJ, USA

X. Wang

Nonclinical Research and Development, Bristol-

Myers Squibb Company, Princeton, NJ, USA

S. Harris

Translational Medicine, Bristol-Myers Squibb

Company, Princeton, NJ, USA on days $5-7,0.92 \mathrm{mg}$ on days $8-10$, and $1.84 \mathrm{mg}$ on days 11-30). On day 30, a single oral dose of PSE $60 \mathrm{mg}$ was co-administered with placebo or ozanimod. Maximum time-matched change in systolic blood pressure (SBP) from baseline (day 29) following PSE administration on day 30 was calculated. Plasma PK parameters for ozanimod, CC112273, CC1084037, and PSE were estimated using noncompartmental methods.

Results: Fifty-two subjects (92.9\%) completed the study. Following multiple dosing, approximately $94 \%$ of circulating total active drug exposure was represented by ozanimod (6\%), CC112273 (73\%), and CC1084037 (15\%). Exposures of CC112273 and CC1084037 were highly correlated. Mean maximum time-matched change from baseline for SBP was not significantly different between ozanimod + PSE and placebo + PSE. Ozanimod also had no effect on the PK of PSE. Co-administration of ozanimod with a single dose of PSE in healthy subjects was generally well tolerated. While CC112273 and CC1084037 selectively inhibited monoamine oxidase (MAO)-B in vitro, both active metabolites do not inhibit platelet MAO$B$ activity in vivo.

Conclusion: Concomitant administration of ozanimod with PSE, a sympathomimetic agent, did not potentiate the effects on blood pressure. Trial Registration: NCT03644576. 
Keywords: Drug

Monoamine

interaction; oxidase;

Pharmacokinetics; Pseudoephedrine

\section{Key Summary Points}

Why carry out this study?

In vitro data showed that ozanimod's major active metabolites, CC112273 and CC1084037, selectively inhibited monoamine oxidase (MAO)-B. MAO inhibitors, when taken in combination with sympathomimetic amines, can cause sudden blood pressure elevations.

We conducted a phase 1 study in healthy subjects to (1) characterize the multipledose pharmacokinetic (PK) properties of CC112273 and CC1084037, (2) evaluate the PK and pharmacodynamic interactions with pseudoephedrine, a sympathomimetic agent, and (3) evaluate the effect of CC112273 and CC1084037 on human platelet MAO-B activity.

\section{What was learned from this study?}

Following multiple dosing, approximately $94 \%$ of circulating total active drug exposure was represented by ozanimod (6\%), CC112273 (73\%), and CC1084037 (15\%).

While CC112273 and CC1084037 selectively inhibited MAO-B in vitro, both active metabolites did not inhibit platelet MAO-B activity in vivo. Concomitant administration of ozanimod with pseudoephedrine did not result in PK or pharmacodynamic interactions.

\section{DIGITAL FEATURES}

This article is published with digital features to facilitate understanding of the article. You can access the digital features on the article's associated Figshare page. To view digital features for this article go to https://doi.org/10.6084/m9. figshare.12918965.

\section{INTRODUCTION}

Ozanimod, a sphingosine 1-phosphate (S1P) receptor modulator that binds with high affinity selectively to $\mathrm{S} 1 \mathrm{P}$ receptors $1\left(\mathrm{~S}_{1} \mathrm{P}_{1}\right)$ and 5 $\left(\mathrm{S}_{\mathrm{P}} \mathrm{P}_{5}\right)$, is approved in the United States for the treatment of adults with relapsing forms of multiple sclerosis (MS) and in the European Union for the treatment of adults with relapsing-remitting MS. Ozanimod blocks the capacity of lymphocytes to egress from lymph nodes, reducing the number of lymphocytes in peripheral blood [1]. The mechanism by which ozanimod exerts therapeutic effects in MS is unknown but may involve the reduction of lymphocyte migration into the central nervous system. Ozanimod is also in clinical development for the treatment of moderate to severe ulcerative colitis and Crohn's disease [2, 3].

Ozanimod is extensively metabolized to form a number of circulating active metabolites, including two major and interconverting active metabolites CC112273 and CC1084037 with similar activity and selectivity for $\mathrm{S}_{1} \mathrm{P}_{1}$ and $\mathrm{S}_{1} \mathrm{P}_{5}$ [4]. Following oral administration of ozanimod single doses of $0.46 \mathrm{mg}$ and $0.92 \mathrm{mg}$ in 56 healthy subjects, ozanimod absorption was slow, with a median time to reach maximum observed concentration $\left(T_{\max }\right)$ of $8 \mathrm{~h}$. Median $T_{\max }$ values for CC112273 and CC1084037 were approximately 10 and $16 \mathrm{~h}$, respectively. Doseproportional increases in exposures were observed for ozanimod, CC112273, and CC1084037. Exposures of CC112273 and CC1084037 also were highly correlated. The mean terminal elimination half-life $\left(t_{1 / 2}\right)$ for ozanimod was approximately $20-22 \mathrm{~h}$ while the mean $t_{1 / 2}$ values for CC112273 and CC1084037 were similar at approximately 10 days [4].

In vitro data showed that CC112273 and CC1084037 selectively inhibited monoamine oxidase (MAO)-B over MAO-A. The half maximal inhibitory concentration $\left(\mathrm{IC}_{50}\right)$ values for $\mathrm{MAO}-\mathrm{B}$ are $5.72 \mathrm{nM}$ and $58 \mathrm{nM}$ for CC112273 and CC1084037, respectively (data on file). The $\mathrm{IC}_{50}$ values for MAO-A are greater than 
$10,000 \mathrm{nM}$ for both major active metabolites. Two isoenzymes of MAO (MAO-A and MAO-B) are present in most mammalian tissues, but the proportions of the two isoenzymes vary from tissue to tissue $[5,6]$. Moreover, some human tissues express only one form of MAO (e.g., fibroblasts and placenta express only MAO-A whereas platelets and lymphocytes express only MAO-B) [7]. MAO catalyzes the oxidative deamination of various monoamines, such as histamine, 5-hydroxytryptamine, and the catecholamines (e.g., dopamine, epinephrine, and norepinephrine) to inactive derivatives [8].

Monoamine oxidase inhibition can lead to peripheral or central neurotransmitter accumulation. Monoamine oxidase inhibitors, when taken in combination with sympathomimetic amines, can cause sudden blood pressure (BP) elevations that may lead to hypertensive crises [9-11]. Pseudoephedrine (PSE), a sympathomimetic agent, is found in many over-thecounter medicines to relieve nasal congestion. The sympathomimetic actions of PSE are primarily the result of norepinephrine release [12]. Inhibition of MAO may reduce the deamination of norepinephrine, leading to pressor effect enhancement of PSE.

This study was conducted to characterize the multiple-dose pharmacokinetic (PK) properties of ozanimod's major active metabolites and to evaluate the effect of ozanimod on the pressor response to a single-dose administration of PSE and on the PK of PSE in healthy adult subjects. The study also evaluated the effect of CC112273 and CC1084037 on human platelet MAO-B activity.

\section{METHODS}

\section{Ethics}

The study protocol and informed consent were reviewed and approved by an institutional review board (IntegReview, Austin, TX, USA; closure date 22 January 2019). All subjects provided written informed consent before study entry. The study was conducted in accordance with the ethical principles of Good Clinical Practice and the Declaration of Helsinki.

\section{Study Population}

Healthy female or male subjects $(N=56)$ aged $18-55$ years, with a body weight of at least $50 \mathrm{~kg}$ and body mass index (BMI) of $18.0-30.0 \mathrm{~kg} / \mathrm{m}^{2}$ were included in the study. Subjects were excluded from the study if they had a seated systolic BP (SBP) outside 90-140 mmHg, seated diastolic BP (DBP) $50-90 \mathrm{mmHg}$, or seated pulse rate outside $55-90 \mathrm{bpm}$; a history of syncope associated with hypotension or a history of orthostatic hypotension; a positive drug/alcohol test; a history of alcoholism, drug abuse, or addiction within 24 months; or a history of any abnormality or illness that, in the opinion of the investigator, may affect absorption, distribution, metabolism, or elimination of the study drugs or would limit the subject's ability to participate in and complete the study. Subjects were prohibited from using any systemic overthe-counter medication (excluding acetaminophen up to $1 \mathrm{~g}$ /day), dietary or herbal supplements within 7 days (28 days for St. John's wort) prior to the first dose of study drug, and any systemic prescription medication (excluding hormonal contraceptives) within 28 days (90 days for MAO inhibitors) or five times the elimination half-life, whichever is longer, prior to the first dose of study drug and during the study.

\section{Study Design}

This was a phase 1, single-center, randomized, double-blind, placebo-controlled study in healthy adult subjects. Subjects were screened for participation within 28 days prior to the first dose of study drug. All eligible subjects were admitted to the clinical research unit (CRU) on day -2 and were domiciled in the CRU until day 31. Eligible subjects were enrolled and randomized 1:1 within either the male or female strata to ozanimod or placebo. Subjects received either placebo or ozanimod once daily (QD) for 30 days $(0.23 \mathrm{mg}$ [equivalent to ozanimod $\mathrm{HCl}$ $0.25 \mathrm{mg}$ ] QD on days 1-4, $0.46 \mathrm{mg}$ [equivalent to ozanimod $\mathrm{HCl} 0.5 \mathrm{mg}$ ] QD on days 5-7, $0.92 \mathrm{mg}$ [equivalent to ozanimod $\mathrm{HCl} 1 \mathrm{mg}$ ] QD on days 8-10, and $1.84 \mathrm{mg}$ [equivalent to 
ozanimod $\mathrm{HCl} 2 \mathrm{mg}$ ] QD on days 11-30). On day 30, a single oral dose of PSE $60 \mathrm{mg}$ was coadministered with placebo or ozanimod. On days 29 and 30, study drug was administered after an overnight fast of at least $10 \mathrm{~h}$ and subjects remained fasted until at least $4 \mathrm{~h}$ after dosing. Meals and mealtimes on days 29 and 30 were also standardized. Following discharge from the CRU on day 31, follow-up safety assessments were conducted at $7 \pm 2$ and $75 \pm 10$ days after the last dose.

\section{PK Blood Sampling Schedule and Bioanalysis}

PK blood samples for ozanimod and its active metabolites were collected at predose and 1, 2, 4, $6,8,10,12,14,16$, and $24 \mathrm{~h}$ postdose on days 1 , 5,8 , and 28 . Trough PK blood samples were collected at predose on days 26,27 , and 30 . PK blood samples for PSE were collected at predose and 1, 2, $4,6,8,10,12$, and $24 \mathrm{~h}$ postdose on day 30 .

Plasma concentrations of ozanimod, major active metabolites (CC112273 and CC1084037), and minor active metabolites (RP101988, RP101442, and RP112289) were determined using the validated liquid chromatography-tandem mass spectrometry method (ICON, Whitesboro, NY). To measure plasma concentrations, ozanimod and its metabolites were extracted from a $400-\mu \mathrm{L}$ aliquot of human $\mathrm{K}_{2}$ ethylenediaminetetraacetic acid plasma by support-liquid extraction and then analyzed using reversed-phase highperformance liquid chromatography with electrospray MS/MS detection. The calibrations were accomplished by weighted linear regressions (1/ $x^{2}$ ) of the ratio of the peak area of the analyte to that of the added deuterated internal standards and plotting to the corresponding nominal concentrations of PSE. The methods were validated over the concentration ranges of $4-2000 \mathrm{pg} / \mathrm{mL}$ for both ozanimod and CC1084037, and $25-10,000 \mathrm{pg} / \mathrm{mL}$ for CC112273. In addition, the assays were validated for the minor metabolites with the concentration ranges of $16-4000 \mathrm{pg} / \mathrm{mL}$ for RP101988, 8-4000 pg/mL for RP101442, and $4-1600 \mathrm{pg} / \mathrm{mL}$ for RP112289. The interassay precision values for ozanimod, CC112273, CC1084037, RP101988, RP101442, and RP112289 were at most 9.8\%. The accuracy values for ozanimod and its metabolites were $-6.7 \%$ to $4.2 \%$.

Plasma concentrations of PSE were determined by using the validated high-performance liquid chromatography-mass spectrometry method (PRA, Lenexa, KS). To measure plasma concentrations, a $50-\mu \mathrm{L}$ aliquot of human dipotassium ethylenediaminetetraacetic acid plasma by protein precipitation was analyzed using reversed-phase high-performance liquid chromatography with electrospray MS/MS detection. The calibrations were accomplished by weighted linear regressions $\left(1 / x^{2}\right)$ of the ratio of the peak area of the analyte to that of the added deuterated internal standards and plotted to the corresponding nominal concentrations of ozanimod and its metabolites, respectively. The methods were validated over the concentration ranges of $0.5-500 \mathrm{ng} / \mathrm{mL}$ for PSE. The interassay precision values (\%CV) for PSE were at most $12.3 \%$. The accuracy (\%bias) values for PSE were within the range of $-3.3 \%$ to $2.8 \%$.

\section{PD Blood Sampling Schedule and Bioanalysis}

Blood samples for the measurement of MAO-B activity in platelets were collected at screening, predose on days $1,5,8,26$ to 30 , and $12 \mathrm{~h}$ postdose on day 1. MAO-B activity in human platelets was measured using the OxiSelect ${ }^{\mathrm{TM}}$ Monoamine Oxidase Assay kit (a simple and sensitive quantitative fluorometric assay for measuring amine oxidase activity in biological samples) and a Biotek Synergy 2 plate reader. Briefly, the assay employs quantitative fluorometric measurement of amine oxidase activity in biological samples. The kit is based on a reaction between MAO-B and its substrate benzylamine that generates $\mathrm{H}_{2} \mathrm{O}_{2}$. Benzylamine is a substrate for MAO-B and semicarbizide-sensitive amine oxidases. The use of MAO-B inhibitor Pargyline distinguishes activity between these enzymes. In the presence of horseradish peroxidase, the fluorometric probe and $\mathrm{H}_{2} \mathrm{O}_{2}$ react to produce resorufin, which in turn is quantified by fluorescence. Activity of MAO-B is then calculated and reported in units per liter. The assay 
was qualified and samples tested by Covance Translational Biomarker Solutions. MAO-B activity ranged from 0.016 to 0.766 units/L. Of the 581 samples received, 577 were within the limits of quantitation of 0.014 to 1.778 units/L.

\section{Cardiovascular, PK, and Safety Assessments}

On days 29 and 30, SBP and DBP were measured in triplicate after subjects were resting for at least $10 \mathrm{~min}$ in a supine position at 0 (predose) and $1,2,3,4,5,6,7,8,10,12$, and $24 \mathrm{~h}$ after dosing. Each of the triplicate measurements was separated by approximately $5 \mathrm{~min}$, and all triplicate measurements were completed within approximately $15 \mathrm{~min}$. The 0 -h triplicate measurements on day 29 were collected within approximately $15 \mathrm{~min}$ before dosing. The $24-\mathrm{h}$ postdose triplicate measurements on day 29 were collected within approximately $15 \mathrm{~min}$ before dosing on day 30, and these measurements were also used as day 30, 0-h measurements. BP at each nominal time point was calculated as a mean of the triplicate measurements.

Pharmacokinetic parameters were calculated using noncompartmental analysis (Phoenix ${ }^{\mathrm{TM}}$ WinNonlin $^{\circledR}$, version 8.0.0.3176) and actual sample collection time. The following PK parameters were estimated for ozanimod and its active metabolites (CC112273, CC1084037, RP101988, RP101442, and RP112289) and PSE: maximum observed concentration $\left(C_{\max }\right), T_{\max }$, and area under the concentration-time curve from time $0-24 \mathrm{~h} \quad\left(\mathrm{AUC}_{0-24}\right)$. Total active $\mathrm{AUC}_{0-24}$ for ozanimod was determined as the sum of ozanimod and all active metabolites $\mathrm{AUC}_{0-24}$, corrected for molecular weight. Plasma concentration values that were below the limits of quantitation (BLQ) were replaced with zero for the mean plasma concentration-time profiles. For PK parameter calculation, BLQ values prior to the first quantifiable value were replaced with zero, and BLQ values after the first quantifiable value were treated as missing.

Physical examinations, 12-lead electrocardiograms (ECGs), vital sign measurements, and clinical laboratory tests were performed. Adverse events (AEs) and concomitant medications were monitored throughout the study to assess safety.

\section{Statistical Evaluation}

No formal hypothesis testing was planned. Using a precision approach, a sample size of 25 each for ozanimod and placebo was expected to produce a two-sided $90 \%$ confidence interval (CI) with a distance from the difference in means to the limits that is equal to 6.64 when the estimated standard deviation (SD) is $14 \mathrm{mmHg}$. Because of potential subject dropout, up to 30 subjects were planned for each treatment group to ensure 25 evaluable subjects within each group.

The primary cardiovascular endpoint was the maximum time-matched change in SBP from baseline (day 29) following PSE administration on day 30. To calculate the time-matched changes for each subject, day 30 value was subtracted from day 29 value at the corresponding nominal time. The maximum of these time-matched changes was computed for each subject. A statistical analysis was performed to compare ozanimod + PSE with placebo + PSE using a linear model with treatment group and sex as fixed effects. The least squares mean (LSM) difference between the two treatment groups and associated 90\% CI were provided.

To estimate the effect of ozanimod on PSE exposure, an analysis of variance was performed for the PSE primary PK parameters $\left(C_{\max }\right.$ and $\left.\mathrm{AUC}_{0-24}\right)$. The primary PK parameters were logtransformed and evaluated in a mixed-effect model with treatment group and sex as fixed effects and subjects as a random effect. Logarithmic differences in LSM of primary PK parameters from PSE co-administered with ozanimod (as test treatment) to PSE co-administered with placebo (as reference treatment) were constructed. Upon back transformation, the PSE geometric LSM ratios of ozanimod over the placebo group and associated 90\% CI were calculated.

The relationship between CC112273 concentrations and change from baseline in 
platelet MAO-B activity was plotted. Similar analysis was performed for CC1084037 concentrations.

The incidence, severity, and relationship of AEs and clinically significant changes from baseline in clinical laboratory tests, vital signs, and ECGs were summarized using descriptive statistics by treatment group. All statistical tabulations and analyses were performed using SAS, version 9.3 (SAS Institute, Cary, NC).

\section{RESULTS}

\section{Subject Disposition and Baseline Characteristics}

Fifty-six subjects were randomized and 52 subjects (92.9\%) completed the study. All subjects in the ozanimod group completed the study. Four subjects $(7.1 \%)$ in the placebo group did not complete the study: two subjects withdrew from the study and two subjects were discontinued from the study because of an AE. Thirtyone (57.4\%) subjects were male and 23 (42.6\%) were female. The mean (SD) age and BMI were 37.5 (11.34) years and $26.11(2.583) \mathrm{kg} / \mathrm{m}^{2}$, respectively. Overall, demographics and baseline characteristics were generally similar between the two treatment groups.

\section{Multiple-Dose PK of Ozanimod and Major Active Metabolites}

The mean (SD) plasma concentration-time profiles for ozanimod and its major active metabolites following dosing initiation on day $1(0.23 \mathrm{mg})$, dose escalation on day 5 (0.46 mg), and on day 28 (1.84 mg) are shown in Fig. 1. Following dosing initiation, the formation of the active metabolites CC112273 and CC1084037 occurred approximately 2 and $4 \mathrm{~h}$ after dosing. Systemic exposure of CC112273 was the highest followed by ozanimod and CC1084037. After 28 days of dosing, systemic exposure of CC112273 remains the highest followed by CC1084037 and then ozanimod. Plasma PK parameters for ozanimod, CC112273, and CC1084037, following dosing initiation on day $1(0.23 \mathrm{mg})$, dose escalation on day 5 (0.46 mg), and after 28 days of repeated dosing $(1.84 \mathrm{mg})$ are summarized in Table 1 . Median $T_{\max }$ was approximately $8-10 \mathrm{~h}$ across dosing days for ozanimod, approximately 10-12 $\mathrm{h}$ across dosing days (approximately $2 \mathrm{~h}$ after ozanimod) for CC112273, and was highly variable (4-24 h) for CC1084037 because of flat PK profiles during the 24-h dosing interval. Upon ozanimod $0.23 \mathrm{mg}$ initiation (day 1), the $\mathrm{AUC}_{0-24}$ values of CC112273 and CC1084037 were approximately twofold and 0.2-fold that of the parent $\mathrm{AUC}_{0-24}$, respectively. Ozanimod, CC112273, and CC1084037 each represented approximately $28 \%, 53 \%$, and $5 \%$, respectively, of circulating total active drug exposure. The metabolite/parent $(\mathrm{M} / \mathrm{P})$ ratios and percentage of total active $\mathrm{AUC}_{0-24}$ for $\mathrm{CC} 112273$ and CC1084037 continued to increase with repeated dosing. On day 28 , the mean $\mathrm{M} / \mathrm{P}$ ratios of the $\mathrm{AUC}_{0-24}$ for CC112273 and CC1084037 were approximately 13-fold and 2.6-fold, respectively. Ozanimod, CC112273, and CC1084037 each represented approximately $6 \%, 73 \%$, and $15 \%$, respectively, of circulating total active drug exposure. Together, ozanimod, CC112273, and CC1084037 contributed to approximately $94 \%$ of circulating total active drug exposure after multiple dosing. The other minor active metabolites (RP101988, RP101442, and RP112289) together contributed to the remaining $6 \%$ of circulating total active drug exposure (data not shown).

Scatter plots with linear regression lines to illustrate the relationship of exposure $\left(C_{\max }\right.$ and $\mathrm{AUC}_{0-24}$ ) between the two major interconverting metabolites CC112273 and CC1084037 are shown in Fig. 2. On day 28, the coefficient of determination $\left(R^{2}\right)$ for $C_{\max }$ and $\mathrm{AUC}_{0-24}$ was 0.976 and 0.979 , respectively. The $C_{\max }$ and $\mathrm{AUC}_{0-24}$ for CC1084037 were approximately $20 \%$ of the $C_{\max }$ and $\mathrm{AUC}_{0-24}$ for CC112273.

\section{Effect of Ozanimod on PSE-Induced Pressor Response}

Table 2 shows the maximum time-matched change from baseline in BP and statistical analysis of the effect of ozanimod on PSE- 
A

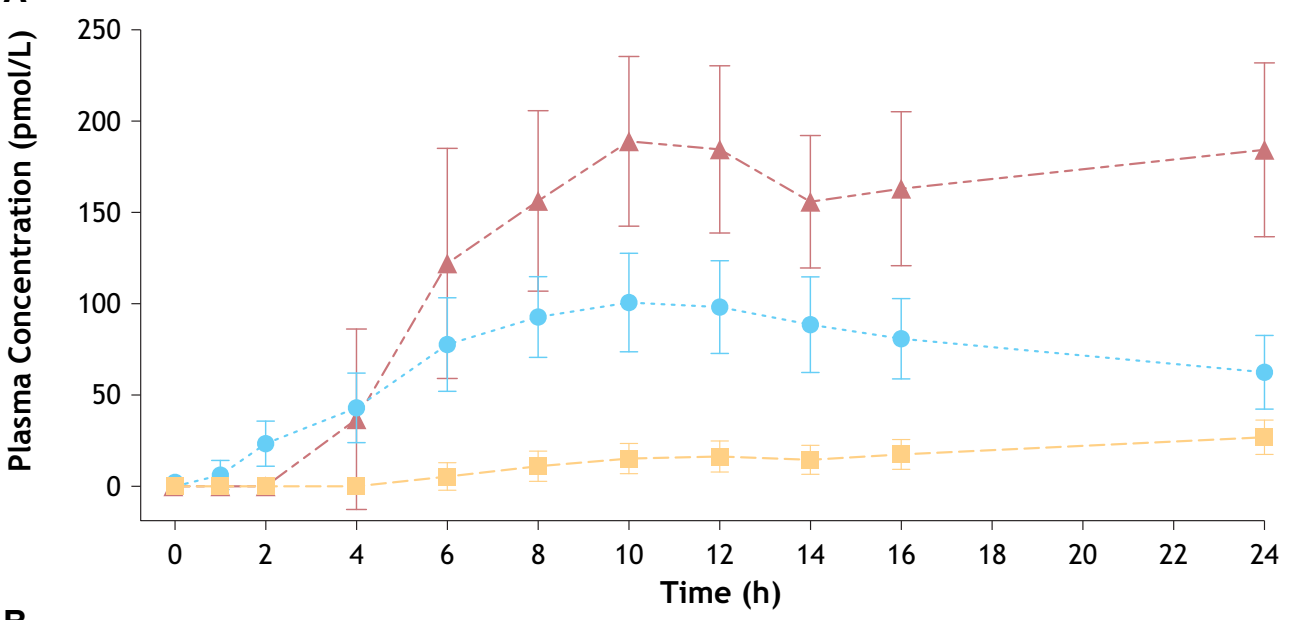

B

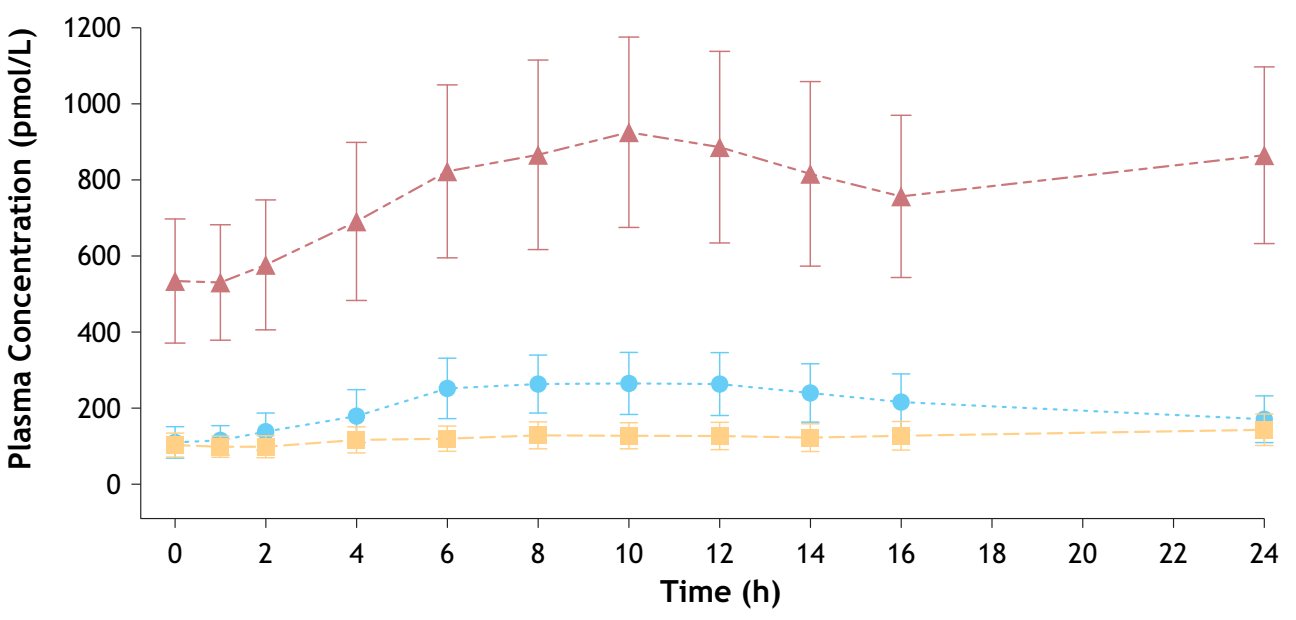

C

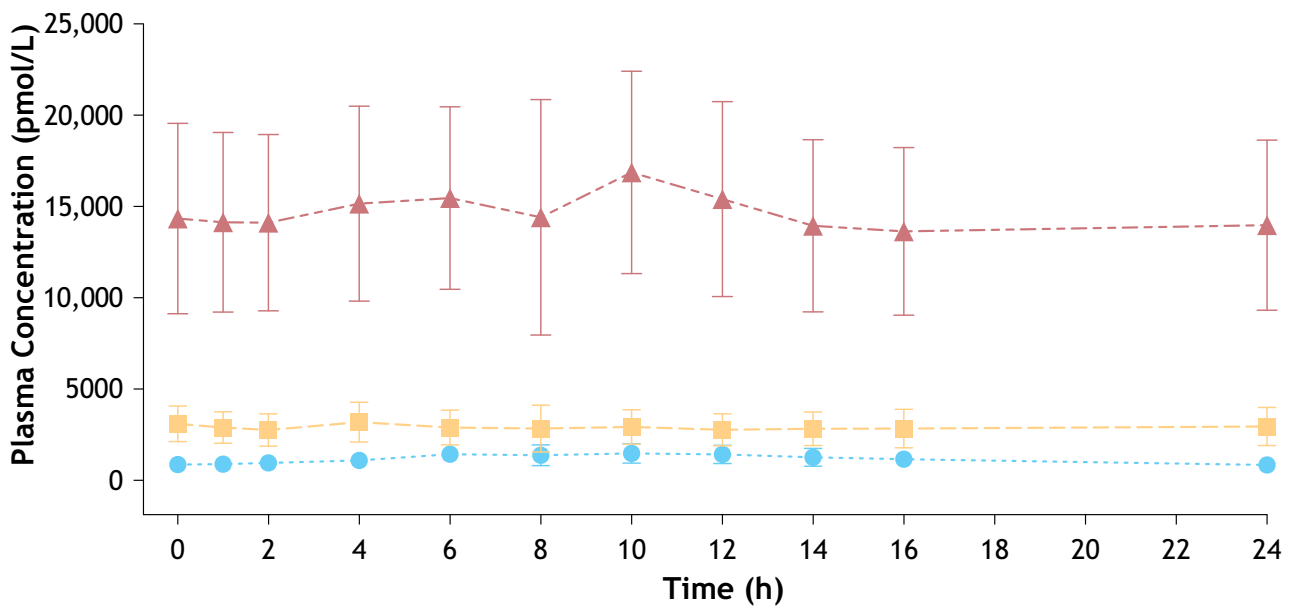

- Ozanimod - - CC112273 - - CC1084037

Fig. 1 Mean (SD) plasma concentration-time profiles for ozanimod, CC112273, and CC1084037 over the 24-h dosing interval following ozanimod dosing initiation on day 1
$(0.23 \mathrm{mg}$; a), dose escalation on day $5(0.46 \mathrm{mg} ; \mathbf{b})$, and after 28 days of repeated dosing $(1.84 \mathrm{mg}, \mathbf{c}) ; N=28$. SD standard deviation 


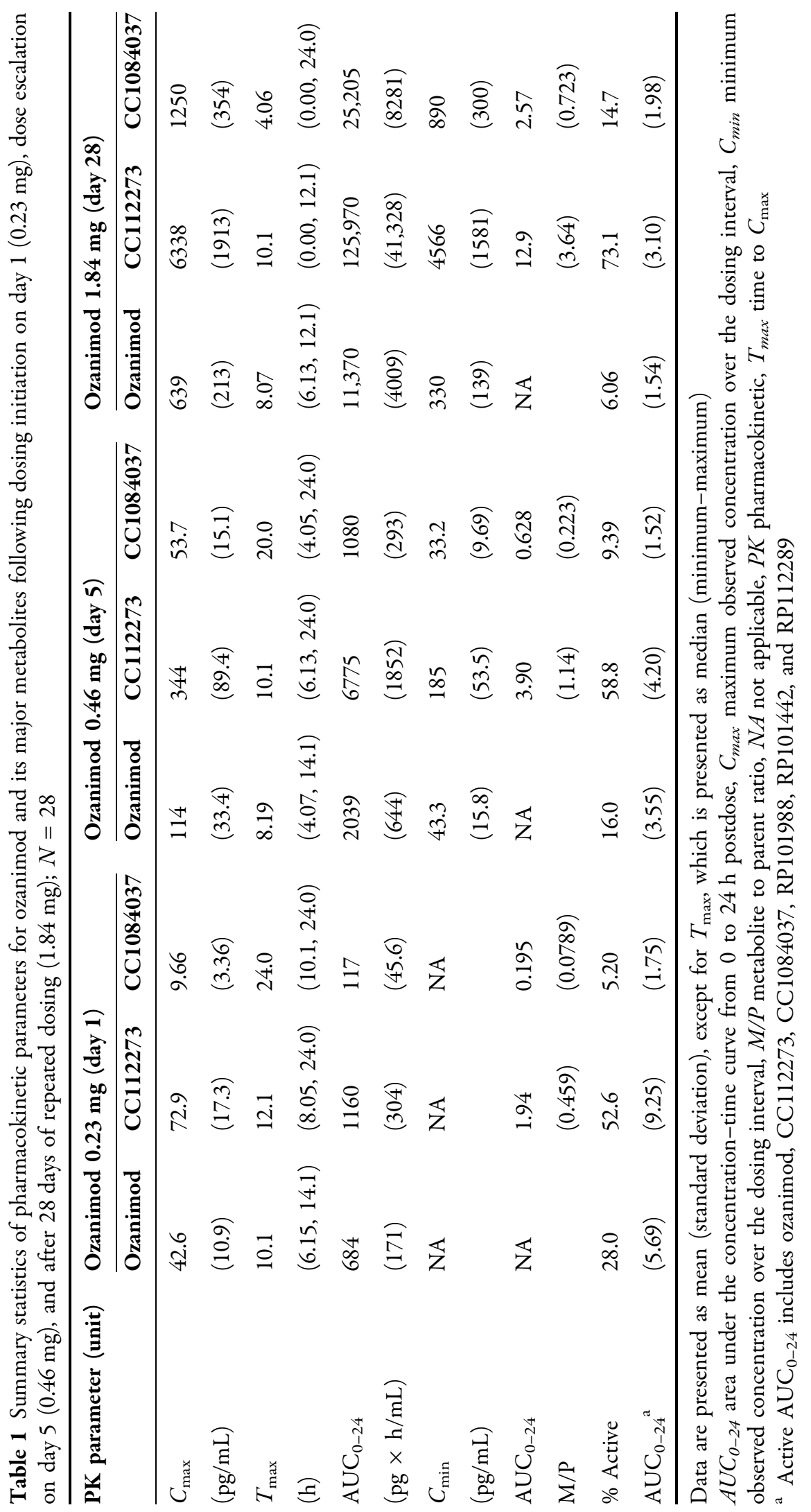



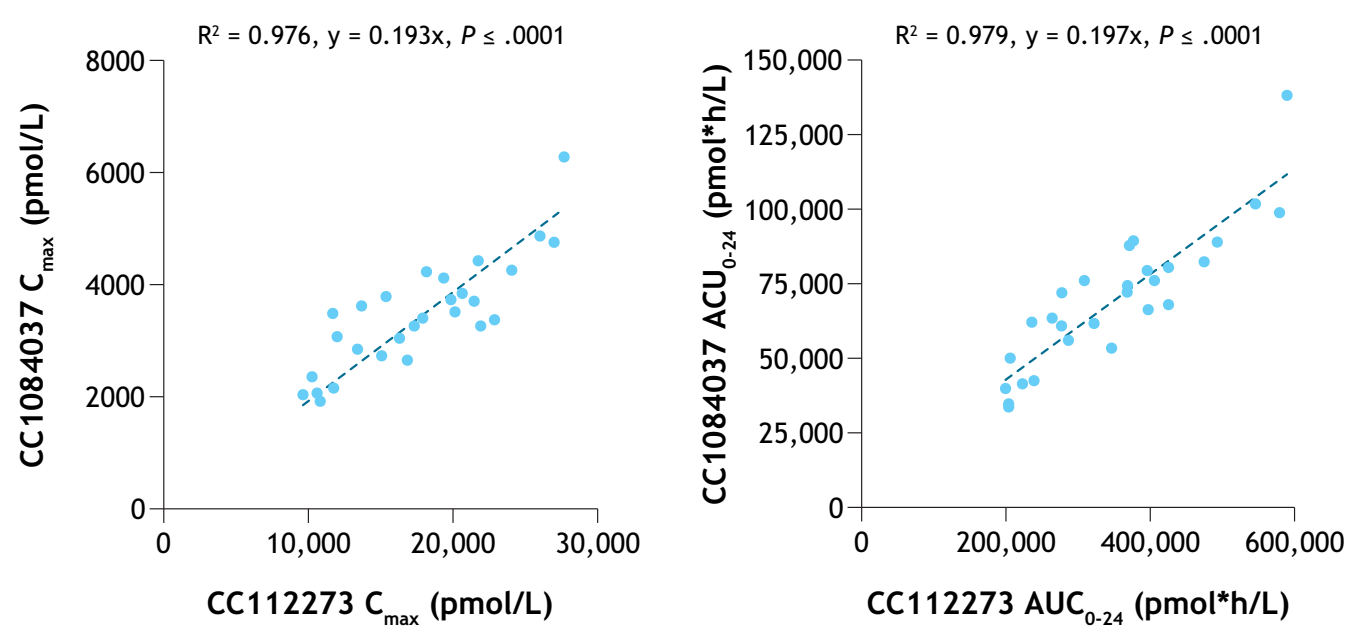

Fig. 2 Relationship of $\mathrm{CC} 112273$ and $\mathrm{CC} 1084037 C_{\max }$ and $\mathrm{AUC}_{0-24}$ on day $28 . \mathrm{AUC}_{0-24}$ area under the concentration-time curve from 0 to $24 \mathrm{~h}$ postdose, $C_{\max }$ maximum observed concentration

Table 2 Effect of ozanimod on PSE-induced pressor response: maximum time-matched change from baseline

\begin{tabular}{|c|c|c|c|c|c|c|}
\hline \multirow{2}{*}{$\begin{array}{l}\text { Cardiovascular } \\
\text { parameters } \\
\text { (units) }\end{array}$} & \multicolumn{2}{|l|}{ Mean (SD) } & \multicolumn{2}{|l|}{ LS means } & \multirow{2}{*}{$\begin{array}{l}\text { LS means } \\
\text { difference } \\
\text { (Test } \\
\text { minus } \\
\text { reference) }\end{array}$} & \multirow{2}{*}{$\begin{array}{l}90 \% \text { CI for LS } \\
\text { means } \\
\text { difference } \\
\text { (Test minus } \\
\text { reference) }\end{array}$} \\
\hline & $\begin{array}{l}\text { Ozanimod + PSE } \\
\text { (Test) } \\
N=28\end{array}$ & $\begin{array}{l}\text { Placebo + } \\
\text { PSE } \\
\text { (Reference) } \\
N=24\end{array}$ & $\begin{array}{l}\text { Ozanimod + PSE } \\
\text { (Test) } \\
N=28\end{array}$ & $\begin{array}{l}\text { Placebo }+ \\
\text { PSE } \\
\text { (Reference) } \\
N=24\end{array}$ & & \\
\hline $\mathrm{SBP}(\mathrm{mmHg})$ & $15.50(5.418)$ & $14.64(5.952)$ & 15.49 & 14.63 & 0.86 & $-1.81,3.53$ \\
\hline $\mathrm{DBP}(\mathrm{mmHg})$ & $9.63(3.683)$ & $8.96(4.444)$ & 9.74 & 9.10 & 0.64 & $-1.23,2.51$ \\
\hline
\end{tabular}

$C I$ confidence interval, $D B P$ diastolic blood pressure, $L S$ least square, $N$ number of subjects, $P S E$ pseudoephedrine, $S B P$ systolic blood pressure, $S D$ standard deviation

induced BP response. PSE co-administered with placebo resulted in a mean maximum timematched change in SBP from baseline of $14.64 \mathrm{mmHg}$. PSE co-administered with ozanimod resulted in a mean maximum time-matched change in SBP from baseline of $15.50 \mathrm{mmHg}$. The maximum time-matched change from baseline for DBP in the ozanimod + PSE group (9.63 $\mathrm{mmHg}$ ) was also similar to the placebo + PSE group $(8.96 \mathrm{mmHg})$. The LSM difference between ozanimod + PSE and placebo + PSE groups was $0.86 \mathrm{mmHg}$ for SBP with a $90 \% \mathrm{CI}$ of $-1.81,3.53 \mathrm{mmHg}$ and $0.64 \mathrm{mmHg}$ for DBP with a $90 \%$ CI of -1.23 , $2.51 \mathrm{mmHg}$.

\section{Effect of Ozanimod on PSE PK}

Mean (SD) plasma concentration-time profiles for a single dose of PSE when co-administered with ozanimod or placebo were superimposable (Fig. 3). Ozanimod had no effect on the exposure of PSE, as demonstrated by the geometric LSM ratios and associated $90 \%$ CIs for $C_{\max }$ $(0.98,0.92-1.04)$ and $\mathrm{AUC}_{0-24}(1.03,0.94-1.13)$ (Table 3).

\section{Effect of Ozanimod on Platelet MAO-B Activity}

There was no relationship between the change from baseline in platelet MAO-B activity and 

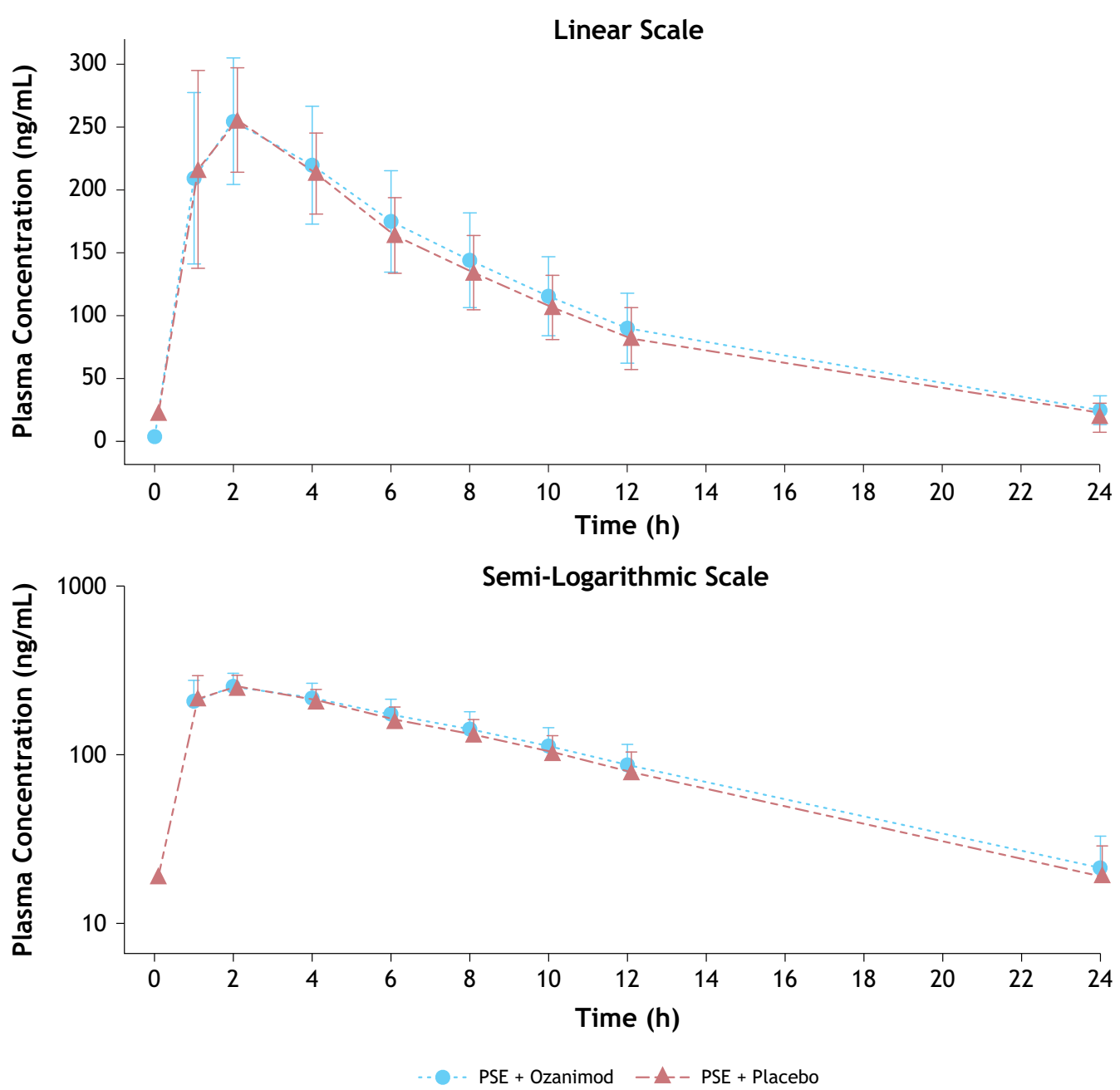

Fig. 3 Mean (SD) PSE plasma concentration-time profiles for a single dose of PSE $60 \mathrm{mg}$ when co-administered with ozanimod or placebo. PSE pseudoephedrine

Table 3 Statistical analysis to assess the effect of ozanimod on a single-dose exposure of PSE $60 \mathrm{mg}$

\begin{tabular}{|c|c|c|c|c|}
\hline \multirow{2}{*}{$\begin{array}{l}\text { PSE primary PK } \\
\text { endpoint (units) }\end{array}$} & \multicolumn{2}{|c|}{ Geometric LS means } & \multirow{2}{*}{$\begin{array}{l}\text { Ratio of geometric LS } \\
\text { means (test to reference) }\end{array}$} & \multirow{2}{*}{$\begin{array}{l}90 \% \text { CI for ratio of } \\
\text { geometric LS means }\end{array}$} \\
\hline & $\begin{array}{l}\text { Ozanimod + PSE } \\
\text { (test) } \\
N=28\end{array}$ & $\begin{array}{l}\text { Placebo }+ \text { PSE } \\
\text { (reference) } \\
N=24\end{array}$ & & \\
\hline$C_{\max }(\mathrm{ng} / \mathrm{mL})$ & 256 & 262 & 0.979 & $0.922,1.040$ \\
\hline $\begin{array}{l}\mathrm{AUC}_{0-24}(\mathrm{ng} \times \mathrm{h} / \\
\mathrm{mL})\end{array}$ & 2450 & 2383 & 1.028 & $0.935,1.130$ \\
\hline
\end{tabular}

$A U C_{O-24}$ area under the concentration-time curve from time 0 to $24 \mathrm{~h}$ postdose, $C I$ confidence interval, $C_{\max }$ maximum observed plasma concentration, $L S$ least square, $P K$ pharmacokinetic, PSE pseudoephedrine

the range of observed plasma CC112273 concentrations that encompassed the mean steady state $C_{\max }\left(C_{\max , \mathrm{ss}}\right)$ for CC112273 of $6980 \mu \mathrm{g} / \mathrm{mL}$ for ozanimod $0.92 \mathrm{mg}$ QD in patients with MS [13] (Fig. 4a). The slope of the regression line was close to 0 with the 95\% CI including 0 . 


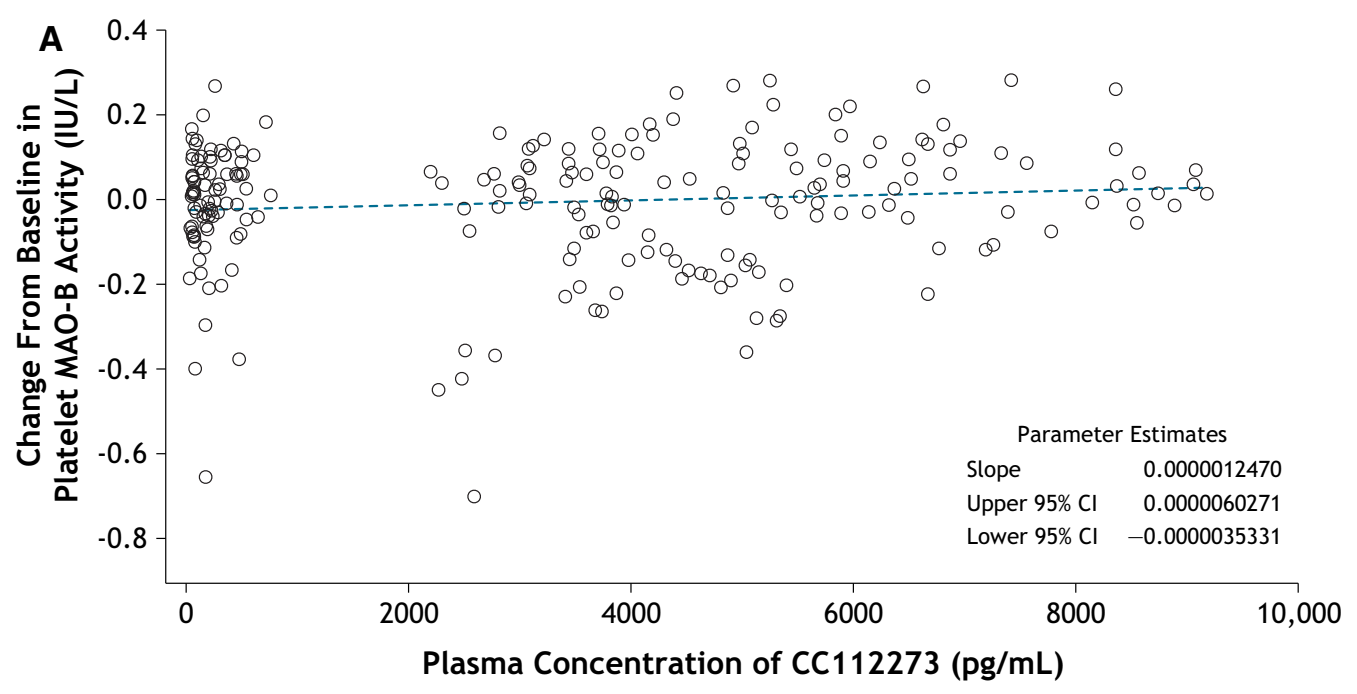

.....-. Regression line

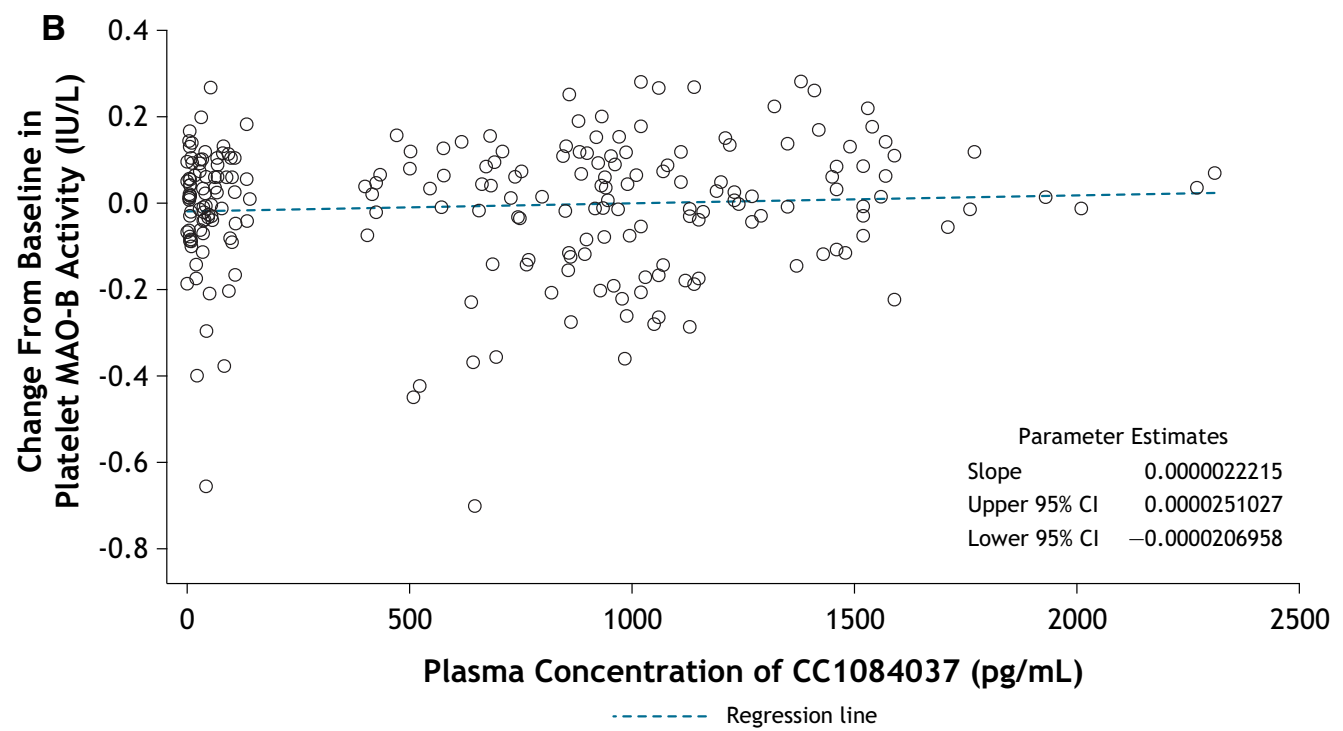

Fig. 4 Change from baseline in platelet's MAO-B activity versus plasma concentration for CC112273 (a) or CC1084037 (b). CI confidence interval, MAO monoamine oxidase

Similarly, there was no relationship between the change from baseline in platelet MAO-B activity and plasma CC1084037 concentrations with the slope of the regression line being close to 0 and the 95\% CI including 0 (Fig. 4b).

\section{Safety}

No serious AEs were reported. Overall, 34 subjects $(63.0 \%)$ had at least one treatment-emergent $\mathrm{AE}$ (TEAE). In the placebo group, 14 subjects $(53.8 \%)$ had at least one TEAE before PSE was co-administered and two subjects (8.3\%) after PSE was co-administered. In the ozanimod group, 19 subjects (67.9\%) had at least one TEAE before PSE was co-administered and four subjects (14.3\%) after PSE was co-administered. The most frequently reported TEAEs in two or more subjects in the placebo treatment group were headache, nausea, vomiting, dizziness, constipation, and musculoskeletal pain. The most frequently reported TEAEs 
reported in two or more subjects in the ozanimod treatment group were headache, nausea, vomiting, constipation, rhinitis allergic, diarrhea, and anemia. The majority of all TEAEs were mild in severity. One subject in the placebo group completed dosing up to day 15 and was discontinued because of abnormal liver function enzymes (alanine aminotransferase [ALT] three times the upper limit of normal [ULN] and gamma glutamyl transferase four times the ULN). One subject in the placebo group was discontinued from the study prior to dosing on day 1 because of an AE of headache. There were no clinically significant changes in vital signs, and no bradycardia or ECG abnormalities. No notable trends for clinical laboratory test results were observed. For examples, the mean (SD) ALT at baseline for placebo and ozanimod groups were 19.8 (11.2) U/L and 16.9 (8.83) $\mathrm{U} / \mathrm{L}$, respectively, and at 7 days after the last dose were 19.5 (10.2) U/L and 21.7 (9.53) U/ $\mathrm{L}$, respectively. The mean $(\mathrm{SD})$ blood urea nitrogen values at baseline for placebo and ozanimod groups were $4.32(0.972) \mathrm{mmol} / \mathrm{L}$ and $4.47(1.23) \mathrm{mmol} / \mathrm{L}$, respectively, and at 7 days after the last dose were $4.43(1.38) \mathrm{mmol} / \mathrm{L}$ and 4.99 (1.66) $\mathrm{mmol} / \mathrm{L}$, respectively.

\section{DISCUSSION}

Ozanimod is approved in the United States for the treatment of adults with relapsing forms of MS and in the European Union for the treatment of adults with relapsing-remitting MS. The recommended maintenance dosage of of ozanimod is $0.92 \mathrm{mg}$ orally QD. Ozanimod may cause a transient decrease in heart rate on dosing initiation. However, this negative chronotropic effect may attenuate over time, secondary to $S 1 \mathrm{P}_{1}$ receptor desensitization, internalization, and degradation on atrial myocytes [14]. An up-titration schedule of ozanimod $0.23 \mathrm{mg}$ QD for 4 days followed by doses of $0.46 \mathrm{mg}$ QD for 3 days prior to the maintenance dose of $0.92 \mathrm{mg}$ attenuates the magnitude of heart rate reduction. To evaluate the potential effect of ozanimod and its major active metabolites on PSE and MAO-B activity, the systemic exposure of CC112273 and
CC1084037 should be similar to the obtained exposure at steady state associated with the therapeutic dose of ozanimod $0.92 \mathrm{mg}$ QD in patients with MS. Considering the $t_{1 / 2}$ of approximately 10 days for both major active metabolites, the required ozanimod dosing would be approximately five times the mean $t_{1 / 2}$ or 50 days, which is not reasonable for healthy subjects. Therefore, ozanimod was titrated to $1.84 \mathrm{mg}$ over 30 days $(0.23 \mathrm{mg}$ QD on days $1-4$, $0.46 \mathrm{mg}$ QD on days 5-7, $0.92 \mathrm{mg}$ QD on days $8-10$, and $1.84 \mathrm{mg}$ QD on days 11-30) in this study to achieve the observed steady-state exposure of CC112273 and CC1084037 in patients with MS receiving the maintenance dose of $0.92 \mathrm{mg}$ QD. A two-way crossover design for drug interaction evaluation is preferred over parallel-study designs. However, as a result of the long $t_{1 / 2}$ of the major active metabolites (approx. 10 days), a crossover study design is not feasible and therefore a parallel-group design was used.

In this study, ozanimod, CC112273, and CC1084037 each represented approximately $28 \%, 53 \%$, and $5 \%$, respectively, of circulating total active drug exposure upon initiation of ozanimod $0.23 \mathrm{mg}$ on day 1 . With repeated dosing, the percentage of total active exposure for CC112273 and CC1084037 continued to increase, which was anticipated because of their long $t_{1 / 2}$. On day 28 , approximately $94 \%$ of circulating total active drug exposure is represented by ozanimod (6\%), CC112273 (73\%), and CC1084037 (15\%). The mean $C_{\max }$ for the major active metabolites on day 28 observed in this study was also similar to the mean steadystate $C_{\max }$ in the patients with MS (data on file). Consistent with the single-dose PK results [4], exposures of CC112273 and CC1084037 were highly correlated following multiple dosing.

MAO inhibitors for the treatment of depression (e.g., phenelzine, tranylcypromine) or Parkinson's disease (e.g., safinamide, rasagiline, selegiline) can interact with sympathomimetic drugs, resulting in sudden BP elevations, which may lead to hypertensive crises. MAO inhibitors are also known to significantly inhibit platelet MAO-B activity [15-17]. Since the major active metabolites of ozanimod selectively inhibited MAO-B in vitro, we conducted the clinical study 
to evaluate the effect of ozanimod treatment on the PSE-induced pressor response. Results showed that ozanimod multiple dosing to achieve steady-state exposure for the major active metabolites associated with the $0.92 \mathrm{mg}$ QD in patients with MS did not potentiate the effect of PSE on BP. The lack of ozanimod treatment effect on the pressor response of PSE could be attributed to the lack of CC112273 inhibitory effect on platelet MAO-B activity.

Certain antibiotics such as linezolid and tedizolid also inhibit MAO in vitro. While linezolid increased the BP response to PSE, tedizolid showed no meaningful interaction with PSE $[18,19]$. One of the potential explanations for the difference in the interaction was the relationship between free-drug concentrations in plasma and the $\mathrm{IC}_{50}$ for MAO inhibition. The tedizolid estimated free $C_{\max }$ was about 4.3-fold and 6.7-fold lower than the $\mathrm{IC}_{50}$ of MAO-A and MAO-B activity, respectively, while the linezolid free $C_{\max }$ was similar to (0.94-fold) and 21-fold higher than $\mathrm{IC}_{50}$ for MAO-A and MAO-B, respectively [19]. On the basis of the plasma protein binding of $99.8 \%$ and $99.3 \%$ for CC112273 and CC1084037, respectively (data on file), free CC112273 and CC1084037 $C_{\max }$ in this study (which are also similar to $C_{\max , \mathrm{ss}}$ in patients with MS receiving ozanimod maintenance dose of $0.92 \mathrm{mg}$ QD) were less than $1 \%$ and $0.001 \%$ of the $\mathrm{IC}_{50}$ for MAO-A and MAO-B activity, respectively. These observations are also consistent with the lack of in vivo effect on human platelet MAO-B activity. However, the clinical relevance of the relationship between free-drug concentrations and $\mathrm{MAO}$ activity needs further confirmation.

\section{CONCLUSIONS}

Following multiple dosing in healthy adults, approximately $94 \%$ of circulating total active drug exposure is represented by ozanimod (6\%), CC112273 (73\%), and CC1084037 (15\%). Exposures of the major and interconverting metabolites CC112273 and CC1084037 were highly correlated. While CC112273 and CC1084037 selectively inhibited MAO-B in vitro, these major active metabolites did not inhibit human platelet MAO-B activity in vivo. Concomitant administration of ozanimod with PSE, a sympathomimetic agent, did not potentiate the effects on BP.

\section{ACKNOWLEDGEMENTS}

We thank the participants of this study.

Funding. This study was sponsored by Celgene Corporation. The Rapid Service and Open Access Fees were funded by Bristol-Myers Squibb Company.

Editorial Assistance. Support for third-party editorial assistance for this manuscript was provided by Lorraine Sweeney of Peloton Advantage, LLC, an OPEN Health company, and was funded by Bristol-Myers Squibb Company.

Authorship. All named authors meet the International Committee of Medical Journal Editors criteria for authorship for this article, take responsibility for the integrity of the work as a whole, and have given their approval for this version to be published.

Disclosures. Jonathan Q Tran is a former employee of Bristol-Myers Squibb Company and a current employee of Mirati Therapeutics. Peijin Zhang, Atalanta Ghosh, Mary Syto, Xiaomin Wang, Sarah Harris, and Maria Palmisano are employees of Bristol-Myers Squibb Company. Susan Walker is an independent consultant (Apex Biostatistics).

Compliance with Ethics Guidelines. The study protocol and informed consent were reviewed and approved by an institutional review board (IntegReview, Austin, TX, USA). All subjects provided written informed consent before study entry. The study was conducted in accordance with the ethical principles of Good Clinical Practice and the Declaration of Helsinki.

Data Availability. Celgene, a Bristol-Myers Squibb Company, is committed to responsible 
and transparent sharing of clinical trial data with patients, healthcare practitioners, and independent researchers for the purpose of improving scientific and medical knowledge as well as fostering innovative treatment approaches. Data requests may be submitted to Celgene, a Bristol-Myers Squibb Company, at https://vivli.org/ourmember/celgene/ and must include a description of the research proposal. The datasets generated during and/or analyzed during the current study are available from the corresponding author on reasonable request.

Open Access. This article is licensed under a Creative Commons Attribution-NonCommercial 4.0 International License, which permits any non-commercial use, sharing, adaptation, distribution and reproduction in any medium or format, as long as you give appropriate credit to the original author(s) and the source, provide a link to the Creative Commons licence, and indicate if changes were made. The images or other third party material in this article are included in the article's Creative Commons licence, unless indicated otherwise in a credit line to the material. If material is not included in the article's Creative Commons licence and your intended use is not permitted by statutory regulation or exceeds the permitted use, you will need to obtain permission directly from the copyright holder. To view a copy of this licence, visit http://creativecommons.org/licenses/by$\mathrm{nc} / 4.0 /$.

\section{REFERENCES}

1. Tran JQ, Hartung JP, Peach RJ, et al. Results from the first-in-human study with ozanimod, a novel, selective sphingosine-1-phosphate receptor modulator. J Clin Pharmacol. 2017;57:988-96.

2. Feagan BG, Sandborn WJ, Danese S, et al. Ozanimod induction therapy for patients with moderate to severe Crohn's disease: a single-arm, phase 2, prospective observer-blinded endpoint study. Lancet Gastroenterol Hepatol. 2020. https://doi.org/10. 1016/S2468-1253(20)30188-6.

3. Sandborn WJ, Feagan BG, Wolf DC, et al. Ozanimod induction and maintenance treatment for ulcerative colitis. N Engl J Med. 2016;374:1754-62.
4. Tran JQ, Zhang P, Surapaneni S, Selkirk J, Yan G, Palmisano M. Absorption, metabolism, and excretion, in vitro pharmacology, and clinical pharmacokinetics of ozanimod, a novel sphingosine 1-phosphate receptor agonist [abstract P993]. In: Triennial Joint Meeting of the European Committee for Treatment and Research in Multiple Sclerosis and Rehabilitation in Multiple Sclerosis; 2019 September 11-13, 2019; Stockholm, Sweden.

5. Shih JC, Chen K, Ridd MJ. Monoamine oxidase: from genes to behavior. Annu Rev Neurosci. 1999;22:197-21717.

6. Tipton KF, Boyce S, O'Sullivan J, Davey GP, Healy J. Monoamine oxidases: certainties and uncertainties. Curr Med Chem. 2004;11(15):1965-82.

7. Shih JC, Chen K. Regulation of MAO-A and MAO-B gene expression. Curr Med Chem. 2004;11: 1995-2005.

8. Youdim MB, Bakhle YS. Monoamine oxidase: isoforms and inhibitors in Parkinson's disease and depressive illness. Br J Pharmacol. 2006;147(suppl 1):S287-S296296.

9. Zyvox [package insert]. New York, NY: Pfizer; 2019 December 2019.

10. Xadago [package insert]. Louisville, KY: US WorldMeds; 2019 November 2019.

11. Zelapar [package insert]. Bridgewater, NJ: Valeant Pharmaceuticals North America; 2016 August 2016.

12. Kobayashi S, Endou M, Sakuraya F, et al. The sympathomimetic actions of L-ephedrine and D-pseudoephedrine: direct receptor activation or norepinephrine release? Anesth Analg. 2003;97: 1239-45.

13. Zeposia [package insert]. Summit, NJ: Celgene Corporation; 2020 March 2020.

14. Brossard P, Scherz M, Halabi A, Maatouk H, Krause A, Dingemanse J. Multiple-dose tolerability, pharmacokinetics, and pharmacodynamics of ponesimod, an S1P1 receptor modulator: favorable impact of dose up-titration. J Clin Pharmacol. 2014;54: 179-88.

15. Müller T, Riederer P, Grünblatt E. Determination of monoamine oxidase A and B activity in long-term treated patients with Parkinson disease. Clin Neuropharmacol. 2017;40:208-11.

16. Thébault JJ, Guillaume M, Levy R. Tolerability, safety, pharmacodynamics, and pharmacokinetics of rasagiline: a potent, selective, and irreversible monoamine oxidase type $B$ inhibitor. Pharmacotherapy. 2004;24:1295-305. 
17. Heinonen EH, Anttila MI, Nyman LM, Pyykkö KA, Vuorinen JA, Lammintausta RA. Inhibition of platelet monoamine oxidase type B by selegiline. J Clin Pharmacol. 1997;37:597-601.

18. Hendershot PE, Antal EJ, Welshman IR, Batts DH, Hopkins NK. Linezolid: pharmacokinetic and pharmacodynamic evaluation of coadministration with pseudoephedrine $\mathrm{HCl}$, phenylpropanolamine
$\mathrm{HCl}$, and dextromethorpan HBr. J Clin Pharmacol. 2001;41:563-72.

19. Flanagan S, Bartizal K, Minassian SL, Fang E, Prokocimer P. In vitro, in vivo, and clinical studies of tedizolid to assess the potential for peripheral or central monoamine oxidase interactions. Antimicrob Agents Chemother. 2013;57:3060-6. 